\title{
Interactive comment on "Hygroscopic growth effect on aerosol light scattering in the urban area of Beijing: a long-term measurement by a wide-range and high-resolution humidified nephelometer system" by P. Zhao et al.
}

\section{Anonymous Referee \#1}

Received and published: 14 September 2018

Review of Zhao et al. (2019) submitted to Atmos Chem Phys.

Summary The paper examines the hygroscopic response of aerosols in the North China Plain in the urban area of Beijing.

The results should generate sufficient interest and provide useful information for the aerosol community. The paper is suitably referenced. The paper needs further work with English and grammar as well as significant rewriting of the analysis to make it more impactful. 
Technical Comments The authors have about 1 month of data for 3 seasons in one year. I would hardly call this a long term measurement campaign as in the title.

I find no obvious technical flaws in assessing the literature and discussing their $\mathrm{f}(\mathrm{RH})$ measurements. However, calibration protocols are not discussed at any length. Some more description of calibration of the nephelometers is merited. Was it $\mathrm{CO} 2$ and clean air? What were the changes over time? The DeBell paper referenced is not a suitable reference for climate effects of aerosols. It's a report on visibility effects.

There were some early measurements of aerosol hygroscopicity in China by Xu, Bergin et al. in the early 2000's that are worth integrating into this paper.

$35 \mu \mathrm{g} / \mathrm{m} 3$ is hard to fathom as 'very clean' conditions. This is the $24 \mathrm{hr}$ PM2.5 standard limit in the US (while the annual standard is 12). I suggest renaming or adding a disclaimer that these definitions are not globally applicable and vary by country. It would be useful to indicate the fraction in time in each of the three PM2.5 levels.

Line 109, I'm not sure that everyone knows the NCP is the most severe area of pollution in China. Does it exceed the Pearl River Delta and other heavily industrialized regions?

'moisture absorption ability' replace with water uptake as aerosols can both absorb and adsorb water. Can you estimate the residence time of the humidified flow with respect to achieving equilibrium? Line 218 Less than $501 / \mathrm{Mm}$ ?

Line 240 discussion of angstrom is hard to follow as to what conditions (season, pollution level etc) dominated when. Suggest a rewrite to make more clear. The discussion of results in lines 300-350 also is somewhat long and hard to comprehend.

Low SSA during clean conditions, to what is this attributed? A large elemental carbon fraction is suggestive of soot from urban-industrial or possibly biomass combustion.

Printer-friendly version

The Monte Carlo simulation of $\mathrm{RH}$ uncertainty is interesting. So how does this relate to the typical $+/-2$ 
Mechanics and Presentation The writing needs a fair bit of work in terms of clarity, grammar and English. The instruments section 2.2 in particular. I cannot give all the polishing needed but will illustrate with a suggested rewriting of the abstract. The abstract really does not summarize the key points of the paper adequately and thus I have rewritten to also improve the English.

"Hygroscopicity is a critical property of ambient aerosols that affects atmospheric light extinction, aerosol radiative forcing of climate, and the formation processes and sinks of aerosols. The light scattering hygroscopic growth factor $(f(R H))$ quantifies this for polydisperse ambient aerosols for continuous relative humidity $(\mathrm{RH})$, typically over the range 20

Line 67 , suggest starting a new paragraph with $f(R H)$.

Line 155, suggest the form here and elsewhere: 'nephelometers (Ecotech, Inc., Model Aurora 3000)' Figure 1, the seasonal panels share the same scale which is helpful, with the exception of the light scattering coefficient time series.

Does it make sense to have a supplement for a single figure? This could be incorporated into the main paper without much 'cost'.

Table 1 units should be specified for scattering and absorption, presumably $1 / \mathrm{Mm}$ The conclusion ends rather abruptly.

Interactive comment on Atmos. Chem. Phys. Discuss., https://doi.org/10.5194/acp-2018-794, 2018. 